\title{
Hexadecadienoic Acid n-7
}

National Cancer Institute

\section{Source}

National Cancer Institute. Hexadecadienoic Acid n-7. NCI Thesaurus. Code C68374.

A polyunsaturated long-chain fatty acid with a 16-carbon backbone and double bonds originating from the 7th position and either the 10th, 11th or 13th positions from the methyl end. At least 3 isomers can be called by this name. 